\title{
General Committee.
}

T. Brain.

Edm. Jones, F.G.S.

T. Lovick.

C. Evans.

T. Dexter.

S. Highley, F.G.S. W. N. Lawson, M.A.

Dr. C. T. Richardson. W. H. Leighton.

J. Jones.

J. Rofe, F.G.S.

J. Noyes.

Honorary Secretary.

J. Cumming, F.G.S., 7, Montague Place, Russell Square, W.C.

Honorary Librarian.

A. Bott, A.A., F.G.S.

Ordinary Meeting, Tuesday, January 5th, 1864.

E. Cresy, President, in the Chair.

A vote of thanks was passed to the President and other officers for their services during the past year.

The following gentlemen were elected members of the Association:-Mr. John Gardner, Mr. Edmund Gardner, and Mr. Robert Irving.

The following donations were announced :-

"Abstract of Proceedings of the Geological Society." By the Society.

A paper was read :-

"On the Fauna of the Carboniferous Epoch." By Professor Morris, F.G.S.

Ordinary Meeting, Tuesday, February 2nd, 1864.

E. Cresy, President, in the Chair.

The following gentleman was elected a member of the Association :-Mr. Edward Cooper. 